Les conditions d'usage des contenus de l'image et des vidéos sont déterminées par les questions de droit dont le numérique a profondément renouvelé l'approche. Après un bref exposé des règles de la propriété littéraire et artistique applicables aux ?uvres audiovisuelles et multimédias, Mélanie Dulong du Rosnay analyse les différentes modalités de gestion des droits : la gestion individuelle par contrat entre le titulaire des droits et l'exploitant, la gestion collective par une société de perception et de répartition des droits, et la gestion numérique à l'aide de mesures techniques de protection et d'information sur les droits comme les DRMs (systèmes de gestion numérique des droits).

\title{
Image et droit, là oủ la technique s'en mêle...
}

LA PRODUCTION ET L'UTILISATION DE banques d'images ou d'œuvres audiovisuelles mettent en jeu des règles de droit qui s'appliquent selon les modalités de la régulation juridique traditionnelle (lois, décisions de justice, contrats, etc.), mais également de manière automatisée par l'intermédiaire de systèmes techniques de protection ou d'information sur les conditions juridiques de diffusion ou d'exploitation.

La propriété littéraire et artistique (PLA) protège dès leur création les images et autres documents audiovisuels à partir du moment où ils obtiennent la qualification juridique d'œuvre de l'esprit. Le Code de la propriété intellectuelle (CPI) accorde cette protection à toute création originale de forme reflétant la personnalité et les choix de l'auteur au-delà d'une simple prestation technique (images fixes et animées, photos analogiques et numériques, cartes géographiques, plans, sites Internet et documents multimédias, etc.), à l'exclusion des idées non mises en forme et des informations brutes qui sont de libre parcours.

Les auteurs et autres titulaires de droits auxiliaires de la création (artistes interprètes, producteurs de phonogrammes, vidéogrammes et bases de données, entreprises de communication audiovisuelle) bénéficient de droits exclusifs qui se traduisent par un monopole temporaire d'exploitation pour toute reproduction, représentation et adaptation de leurs œuvres. L'obtention de leur 
autorisation écrite préalable dans un contrat sera nécessaire, sauf dans certains cas prévus par la loi : les œuvres du domaine public, les exceptions et limitations aux droits exclusifs.

Les Digital Rights Management systems (DRMs) ou systèmes de gestion numérique des droits permettent de rétablir artificiellement la rareté et l'exclusivité de biens dont la production, la reproductibilité et la large diffusion ne rencontrent plus d'obstacle financier ou technique. Certains reprochent à ces DRMs de porter atteinte aux droits des utilisateurs : l'architecture de la plupart de ces systèmes interdit l'exercice de la copie privée, et le traitement des données personnelles de consommation menace la vie privée. Enfin, ces technologies propriétaires développées par des entreprises ou des consortiums industriels de standardisation technique ne sont pas toujours interopérables: elles ne peuvent pas être exécutées sur toutes les plates-formes, systèmes d'exploitation et lecteurs multimé-
Mélanie Dulong de Rosnay

(IEP Lyon 1996) a étudié le droit international et européen à Lyon, Leipzig et Tilburg. Elle prépare une thèse de droit au Centre d'études et de recherches de science administrative (CERSA CNRS/Université Panthéon-Assas

Paris-II), où elle est responsable juridique du projet de contrats d'accès ouvert Creative Commons France. Elle a coordonné des projets culturels et de recherche, notamment à l'IRCAM, enseigné la propriété littéraire et artistique à l'Université de technologie de Compiègne et participe à des projets de recherche sur les ontologies juridiques, la gouvernance et les standards du multimédia. melanie.dulong-de-rosnay@cersa.org dias, ce qui conduit à une fragmentation du marché dommageable pour les utilisateurs.

Après une brève présentation des règles de la PLA applicables aux œuvres audiovisuelles et multimédias, nous analyserons les différentes modalités de gestion des droits : la gestion individuelle par contrat négocié entre le titulaire des droits et l'exploitant, la gestion collective par l'intermédiaire d'une société de perception et de répartition des droits, et la gestion numérique à l'aide de mesures techniques de protection et d'information sur les droits.

\section{Le statut juridique} des œuvres audiovisuelles et multimédias

La qualification juridique des œuvres définit la titularité des droits, et il importe de bien retracer les différents apports et contributions afin de déterminer les autorisations à négocier et les prérogatives dont les ayants droit pourront se prévaloir. Les règles applicables aux ouvres plurales (réalisées par plusieurs personnes) dépendent de la manière dont ces œuvres ont été créées. Il est utile de conserver les traces du processus de la création (courriers électroniques, comptes rendus de réunions, etc.) qui pourront contribuer à la formation d'un faisceau d'indices déterminant la titularité des droits exclusifs.

Les œuvres plurales

Si les intervenants ont travaillé en même temps, il s'agit de savoir si plusieurs personnes physiques ont agi en se concertant et dans un but commun tout au long de la réalisation de la création. Dans ce cas, l'accord unanime de tous les participants sera nécessaire pour exploiter l'œuvre dite « de collaboration ». Chaque co-auteur conservera la possibilité d'exploiter séparément sa contribution, si les différents apports des auteurs relèvent de genres différents et que cela ne nuit pas à l'exploitation de l'œuvre commune.

Sera qualifiée d'" œuvre collective », en revanche, "l'œuvre créée sur l'initiative d'une personne physique ou morale qui l'édite, la publie, la divulgue sous sa direction et son nom et dans laquelle la contribution personnelle des divers auteurs participant à son élaboration se fond dans l'ensemble en vue duquel elle est conçue, sans qu'il soit possible d'attribuer à chacun d'eux un droit distinct sur l'ensemble réalisél ${ }^{1}$. Ici, le donneur d'ordre ou coordinateur (l'employeur de créateurs salariés ou fonctionnaires) sera la seule personne investie initialement des droits, sans qu'un contrat de cession soit nécessaire en complément du contrat de travail ou de commande.

Ainsi une bande dessinée, une chanson ou un opéra sont typiquement des œuvres de collaboration alors qu'une encyclopédie ou un journal sont des œuvres collectives.

La loi peut également raisonner selon la nature de l'œuvre : une catégorie distincte est réservée aux bases de données (voir en fin de section), et elle assimile les logiciels à des œuvres collectives, et les œuvres audiovisuelles à des œuvres de collaboration, avec présomption de cession des droits d'exploitation au producteur' ${ }^{2}$.

Néanmoins, la jurisprudence révèle la fragilité de cette distinction, notamment pour les œuvres multimédias ${ }^{3}$, où l'on voit s'opposer deux logiques : la logique économique plaidera pour une qualification d'œuvre collective, et la logique d'auteur sera en faveur du rattachement au statut d'œuvre de collaboration. Une distinction claire entre ces deux catégories d'œuvres plurales est essentielle, parce que l'attribution des droits et la sécurité juridique sont des conditions nécessaires préalables à tout investissement humain et financier. Le statut de l'œuvre multimédia fait l'objet de débats doctrinaux particulièrement vifs, la partie logicielle n'étant pas essentielle, et l'aspect interactif dépassant la définition d'œuvre audiovisuelle. Les définitions légales et prétoriennes doivent être interprétées au cas par cas, tout contrat étant susceptible d'être « requalifié » par le juge. 
Si les contributeurs ont travaillé de manière successive, on se trouvera face à une œuvre composite, définie comme «l'œuvre nouvelle à laquelle est incorporée une ouvre préexistante sans la collaboration de l'auteur de cette dernière ${ }^{4} »$, par exemple une traduction, une anthologie, une adaptation... Lauteur de l'œuvre composite sera titulaire des droits sur l'œuvre nouvelle, sans préjudice des droits de l'auteur de l'œuvre originaire dont l'autorisation sera nécessaire avant l'exploitation de l'œuvre composite.

Naturellement, ces statuts peuvent se superposer : ainsi l'adaptation d'une œuvre audiovisuelle en jeu vidéo pourra être qualifiée à la fois d'œuvre collective et d'œuvre composite.

De même, un document audiovisuel combine bien souvent des droits d'auteur et des droits voisins. Introduits en 1985 dans la PLA, les droits voisins protègent les auxiliaires de la création pendant cinquante ans après la fixation ou l'exécution de l'œuvre: l'autorisation des artistes interprètes, des producteurs de phonogrammes ou de vidéogrammes responsables de la première fixation d'une séquence de son ou d'images et, le cas échéant, des entreprises de communication audiovisuelle (radios, télévisions) sera requise avant toute exploitation publique. Depuis 1996, le producteur de bases de données se voit reconnaître des droits exclusifs sur l'extraction et la réutilisation substantielles du contenu de la base, indépendamment des droits d'auteur et des droits voisins portant sur les éléments constitutifs de la base de données, définie par le CPI comme « un recueil d'œuvres, de données ou d'autres éléments indépendants, disposés de manière systématique ou méthodique, et individuellement accessibles par des moyens électroniques ou par tout autre moyen ${ }^{5}$.

\footnotetext{
${ }^{1}$ Article L. 113-2 du CPI.

2 Article L. 132-24 du CPI.

${ }^{3}$ Judith Andrès et Pierre Sirinelli, Rapport sur les aspects juridiques des œuvres multimédias, étude du Centre d'études et de recherche en droit de l'immatériel commandé par le Conseil supérieur de la propriété littéraire et artistique du ministère de la Culture et de la Communication, 2003. www.culture.gouv.fr/culture/cspla/Aspectsjuridiquesdesoeuvr esmultimedias.pdf

${ }^{4}$ Article L. 113-2 du CPI.

${ }^{5}$ Article L. 112-3 alinéa 2 du CPI.

${ }^{6}$ Voir les articles L. 122-5, L. 211-3, L. 214-1, L. 342-3 du CPI. 7 Directive 2001/29/CE du Parlement européen et du Conseil du 22 mai 2001 sur l'harmonisation de certains aspects du droit d'auteur et des droits voisins dans la société de l'information, ]0 L 167 22.06. 2001, p. 10.

http://europa.eu.int/eurlex/pri/fr/oj/dat/2001/l_167//_16720010622fr00100019.pdf

${ }^{8}$ Question écrite $n^{\circ} 70276$, www.assemblee-

nationale.fr/12/tribun/fiches_id/1871.asp
}

Les droits moraux

Aux droits patrimoniaux mis en œuvre en cas d'exploitation publique de l'œuvre (reproduction, communication au public, adaptation, vente, location, etc.) s'ajouteront pour les personnes physiques, auteurs et artistes interprètes, des prérogatives d'ordre moral. Les droits moraux comprennent le droit de paternité, le droit de divulgation, le droit de retrait et le droit au respect de l'œuvre. Les liens hypertextes facilitent l'application du droit au respect du nom et de la qualité de l'auteur. Mais la numérisation et les logiciels de compression renouvellent les problématiques liées au droit au respect, les transformations pouvant porter atteinte à l'intégrité de l'œuvre ou à la réputation de l'auteur.

\section{Le droit à l'image}

D'autres éléments sont susceptibles de donner lieu à une protection additionnelle à la PLA dans le cas de la reproduction photographique : le droit à l'image des personnes et de leurs biens, la protection de produits par dessins ou modèles, ou encore le droit des marques. Les récents revirements de jurisprudence entre propriétaires et photographes révèlent qu'une autorisation sera nécessaire si le bien reproduit constitue le sujet principal de la photographie et que le propriétaire apporte la preuve d'un trouble du droit d'usage ou de jouissance. De même, l'utilisation d'œuvres architecturales ou de sculptures situées dans des lieux publics donnera prise à des droits exclusifs si l'œuvre est le sujet principal de la reproduction. L'exploitation de données personnelles, incluant l'image des personnes, nécessite leur consentement écrit, et leur traitement doit faire l'objet d'une déclaration à la Commission nationale de l'informatique et des libertés (CNIL). La jurisprudence révèle que le droit à l'image des personnes et des biens, qu'ils relèvent ou non du droit d'auteur, tolère peu d'exceptions (illustration immédiate d'un sujet d'actualité, reproduction accessoire, non identifiable ou inévitable).

\section{Les exceptions et limitations aux droits exclusifs}

Les exceptions aux droits de $\mathrm{PLA}^{6}$ sont plus larges, les titulaires de droits ne peuvent s'opposer aux représentations et copies privées, aux parodies et caricatures, aux courtes citations, aux reproductions pour les besoins du dépôt légal, etc. Le législateur français pourrait profiter de la transposition en cours de la directive européenne de 2001 sur le droit d'auteur ${ }^{7}$ pour ajouter une exception en faveur de l'enseignement, de la recherche, des bibliothèques, musées et archives; mais il ne saisira apparemment pas cette opportunité ${ }^{8} \ldots$

Lexception de copie privée est remise en question par le droit communautaire qui autorise les États à limiter cette prérogative du public dans 
le cas de services interactifs en ligne. Le droit français prévoit une liste limitative, à l'inverse de la méthode des pays de common law qui propose des critères d'interprétation des cas d'usage équitable (fair use aux États-Unis, fair dealing au RoyaumeUni). La durée des droits exclusifs est un autre facteur qui diffère selon les juridictions. En Europe, l'œuvre tombe (ou s'élève) dans le domaine public soixante-dix ans (plus années de guerre) après la mort du dernier coauteur, cinquante ans après la fixation de la séquence d'image ou sa première communication au public pour les droits voisins, et quinze ans après l'achèvement d'une base de données, avec une reconduction de cinq ans en cas de nouvel investissement substantiel.

Le documentaliste sera libre de réaliser des résumés bibliographiques à la condition que la consultation de ceux-ci ne dispense pas de la lecture de l'œuvre protégée' ${ }^{9}$. Les résumés ainsi que les métadonnées, le thésaurus et le système d'indexation d'une base de données bibliographique sont protégés par le droit des producteurs de bases de données, soit en général l'institution qui prend l'initiative et le risque des investissements.

À propos d'un reportage télévisé sur une exposition consacrée à Maurice Utrillo, la cour d'appel de Paris a déclaré que la représentation intégrale d'une œuvre, quelle qu'en soit la forme ou la durée, ne peut relever de l'exercice du droit de citation ni être considérée comme accessoire, et l'invocation du droit à l'information énoncé dans l'article 10 de la Convention européenne des droits de l'homme a été rejetée par la Cour de cassation ${ }^{10}$.

Certaines utilisations donnent lieu à une licence légale et à une gestion collective obligatoire (la gestion collective demeure facultative en dehors de ces cas). Il n'y a pas d'autorisation préalable à demander, mais une rémunération dite " équitable » à verser à la société de perception et de répartition des droits compétente, pour le droit d'auteur dans le cas de la reproduction par reprographie et du prêt public d'ouvrages en bibliothèques, et pour les droits voisins dans les cas de radiodiffusion, de communication au public de phonogrammes, ainsi que de diffusion simultanée par câble ou satellite de phonogrammes et vidéogrammes télédiffusés. Un projet de $\operatorname{loi}^{11}$ a été déposé pour étendre la licence légale aux échanges de fichiers protégés sur des services de communication en ligne par des particuliers à des fins non commerciales. Les représentants des sociétés d'auteurs et des producteurs de phonogrammes, désireux de conserver un droit exclusif, et les fournisseurs d'accès qui seraient en charge de la perception de cette rémunération sont opposés à cette proposition soutenue par les représentants des artistes interprètes et des consommateurs.

Les titulaires de droits qui le souhaitent ont à leur disposition des contrats types pour autoriser le public à exercer gratuitement et sans négociation préalable plus de droits que la loi ne le permet : il s'agit du « domaine public consenti » ou du renoncement à l'avance à exercer certaines prérogatives par l'intermédiaire de licences libres ou contrats d'accès ouvert. Aux côtés de ces outils juridiques, diverses initiatives ${ }^{12}$ en faveur de l'accès ouvert à la connaissance soutiennent le développement de plates-formes d'auto-archivage des articles scientifiques et de revues alternatives en libre accès.

En dehors des exceptions et limitations légales et contractuelles, toute exploitation d'une ouvre devra donner lieu à une autorisation dans un contrat écrit qui mentionnera l'étendue des droits cédés, la destination, le lieu et la durée. La rémunération est en principe proportionnelle aux recettes. Linterprétation des contrats est restrictive, il importe de décrire avec précision l'étendue de chacun des droits cédés : toute exploitation sous forme non prévisible doit comporter une participation aux profits d'exploitation et tout droit dont l'étendue n'aurait pas été décrite de manière suffisamment précise sera réputé non cédé.

\section{Les mesures techniques de protection et d'information}

Linnovation, par la conjonction des réseaux et des techniques de compression et de reproductibilité, facilite la diffusion des œuvres. Elle permet aussi de développer de nouveaux modes de régulation: des mesures techniques de protection des œuvres et des mesures techniques d'information sur les droits sont développées contre les effets non désirés par certains ayants droit de la facilité de diffusion et de reproduction des fichiers numériques.

\section{La protection juridique des mesures techniques}

La mise en place de systèmes techniques visant à limiter la reproduction d'œuvres, ou l'accès à certaines d'entre elles, ainsi qu'à favoriser leur identification et leur traçabilité est soutenue par les industries du contenu, les fabricants de matériel électronique et informatique, les représentants des ayants droit et les programmes de subvention de la recherche. L'efficacité de ces dispositifs a été reconnue par le droit international en 1996 avec deux conventions de l'OMPI (Organisation mondiale de la propriété intellectuelle). La directive européenne en cours de transposition en droit fran- 
çais $^{3}$ contraint les États membres à mettre en place une protection juridique contre le contournement des mesures techniques de protection des œuvres et d'information sur le régime des droits : le droit protège les techniques qui protègent les droits contre la technique...

Lors de la mise en place de tels dispositifs, une attention particulière doit être accordée à la compatibilité entre la protection technique et le respect d'autres intérêts. Les États européens doivent adopter des «mesures appropriées » pour assurer que les bénéficiaires d'exceptions puissent exercer leurs prérogatives dès l'instant où ils disposent d'un accès licite à l'œuvre, néanmoins la directive ne précise pas le contenu desdites mesures. Le projet de loi français prévoit ${ }^{13}$ l'installation d'un « collège de médiateurs » auquel seront soumis les différends portant sur le bénéfice des exceptions aux droits exclusifs et qui impliquent une mesure technique. La définition légale d'une mesure technique « efficace » est très délicate. Il importe en effet de ne pas porter atteinte à d'autres droits, et de préserver les innovations qui pourraient incidemment conduire à contourner une mesure technique sans que ce soit l'objectif principal : choix du logiciel de lecture, interopérabilité, archivage par les bibliothèques, compression et autres traitements numériques du signal audiovisuel, recherche en cryptographie, mais également la vie privée, le droit de la concurrence, les pratiques de lecture et de citation, etc.

\section{Les mesures techniques d'identification}

Différentes techniques sont disponibles pour s'assurer du respect des droits et contrôler les utilisations. Les méthodes de cryptographie et de stéganographie permettent de contrôler l'accès et la copie, de brouiller le flux audiovisuel ou encore d'ajouter des informations invisibles dans les

\footnotetext{
${ }^{9}$ Cour de cassation, Assemblée plénière, 30 octobre 1987, décision « Microfor ». www.foruminternet.org/documents/general/lire.phtml?id=236 ${ }_{10}$ Cour d'appel de Paris, $4{ }^{\mathrm{e}}$ chambre, section A, 30-05-2001; Cour de cassation, Chambre civile 1, 13-11-2003.

${ }^{11}$ www.assemblee-nationale.fr/12/propositions/pion2474.asp

12 Initiative de Budapest pour l'Accès Ouvert, 14 février 2002, Déclaration de Berlin sur le libre accès à la connaissance en sciences exactes, sciences de la vie, sciences humaines et sociales du 22 octobre 2003.

${ }^{13}$ Article 9 du projet de loi relatif au droit d'auteur et aux droits voisins dans la société de l'information. www.culture.gouv.fr/culture/actualites/communiq/aillagon/dr oitdauteur1103.pdf

${ }^{14}$ www.iddn.org

${ }^{15} \mathrm{http}: / /$ fr.creativecommons.org

${ }^{16}$ Danièle Bourcier et Mélanie Dulong de Rosnay, « La création comme bien commun universel - Réflexions sur un modèle émergent ». In : International Commons at the Digital Age - La création comme bien commun à l'ère du numérique. 2004. http://fr.creativecommons.org/iCommons_book.htm
}

fichiers numériques. Également, les technologies d'association persistante ou de simples liens hypertextes permettront de s'assurer que les informations sur les droits resteront attachées à une œuvre.

Des normes d'identification unique des œuvres développées au sein de l'ISO (Organisation internationale de normalisation) sont administrées par la CISAC (Confédération internationale des sociétés d'auteurs et de compositeurs) :

- l'ISAN (International Standard Audiovisual Number) pour les œuvres audiovisuelles;

- l'ISRC (International Standard Recording Code) pour les enregistrements sonores et de vidéo de musique;

- l'ISWC (International Standard Musical Work Code) pour les œuvres musicales.

Ces identifiants uniques sont utilisés pour la répartition de droits, mais ils peuvent aussi servir pour mener des opérations de contrôle des œuvres diffusées et pour identifier d'éventuelles contrefaçons, ou encore réaliser des mesures d'audience.

Les techniques d'aquamarquage (watermarking) et de filigrane permettent également d'assurer le suivi des œuvres. Elles facilitent l'application du droit puisque la copie ou l'œuvre dérivée non autorisées porteront la marque théoriquement inamovible de l'œuvre originale; elles manquent cependant de robustesse face aux traitements du signal. Plutôt que d'insérer un identifiant, il est également possible de calculer automatiquement à partir du signal numérique des signatures qui identifieront toutes les œuvres, même celles qui n'ont pas été marquées ou dont le marquage aurait été retiré.

D'autres mesures techniques cherchent au contraire à être visibles par tous les utilisateurs, c'est le cas des mesures d'information sur les droits. Le système IDDN (International Identifier of Digital Works ${ }^{14}$ ) permet aux titulaires de droits d'enregistrer leur œuvre en apposant une signature électronique et en délivrant un certificat. Ce dépôt pourra être constitutif d'une preuve d'antériorité en cas de conflit. Il référence pour chaque œuvre les informations sur la titularité des droits et les conditions d'exploitation par l'intermédiaire d'un lien à placer sur la page html présentant l'œuvre. Plus médiatisé, le générateur de contrats types Creative Commons ${ }^{15}$ propose d'apposer des métadonnées juridiques aux œuvres, également grâce à un lien hypertexte vers des licences de libre diffusion plus ou moins restrictives : le titulaire de droits a la possibilité d'autoriser à l'avance l'exercice de certains droits comme l'exploitation commerciale ou la création d'œuvres dérivées, ou au contraire de réserver certains de ces droits. Conformément aux principes du web sémantique, Creative Commons ne détient pas de base de données centralisée, n'enregistre pas les œuvres, et fournit des outils juridiques et techniques pour accéder plus facilement aux créations et 
encourager la culture et la science libre, vers la constitution de biens communs partageables par tous ${ }^{16}$.

Les mesures techniques de contrôle d'accès et les DRMs

De nombreux systèmes de cryptage rendent impossible la lecture ou la copie du fichier et permettent de contrôler l'accès à l'œuvre par l'intermédiaire de clés ou de mots de passe. La protection peut être mise en œuvre par l'intermédiaire du lecteur, d'une carte à puce ou du support physique, ou être intégrée directement à l'intérieur du fichier numérique. Elle peut encore combiner ces différentes technologies, comme la solution Medialive qui propose de brouiller les œuvres audiovisuelles en modifiant certaines données, les rendant imperceptibles aux sens humains.

Les DRMs, systèmes de DRM ou gestion numérique des droits, associent les fonctionnalités d'identification et de contrôle d'accès avec le rapport des utilisations effectives et la répartition des sommes collectées entre les différents ayants droit. Les rights de DRM ne correspondent pas nécessairement à des « droits », des prérogatives issues de droits exclusifs. Cette expression ne désigne pas des prérogatives juridiques, mais au contraire des modèles économiques, des conditions de vente mises en œuvre par des systèmes informatiques. Ainsi, un système de DRM pourra offrir une prévisualisation gratuite, moduler la tarification et le nombre de lectures ou de copies ultérieures, et contrôler le respect de ces conditions, le paiement et la gestion des données d'utilisation.

\section{La normalisation des interfaces}

Les DRMs intègrent différents modules logiciels aux fonctions multiples : identification, description et transmission des fichiers, authentification des utilisateurs, langages d'expression et dictionnaires des droits, construction d'une architecture pour la protection et la distribution sécurisée, mise en place de systèmes de répartition des rémunérations et de rapport des utilisations, etc. L'effectivité de ces systèmes de DRM suppose leur normalisation en vue d'assurer la compatibilité des modules avec les différents formats, lecteurs multimédias et matériels informatiques. Les organismes de normalisation technique requièrent en principe que les titulaires de brevets sur des éléments de la norme accordent une licence d'utilisation selon des conditions raisonnables et non discriminantes à toute personne qui souhaiterait implémenter les spécifications de cette norme dans un produit ou un logiciel. On ne confondra pas le terme de standard ouvert, qualificatif employé par de nombreux consortiums pour indiquer que leur standard peut être intégré dans différentes applications contre une rémunération, avec le principe open source et les logiciels libres qui garantissent l'accès au code source.

Le sous-comité de l'ISO dédié au codage du multimédia et des images fixes prépare les normes MPEG, Moving Picture Expert Group, et JPEG, Joint Picture Expert Group. Lindustrie et la recherche académique travaillent de manière volontaire et consensuelle pour élaborer des standards de compression et de transmission de l'information numérique, et intègrent les questions de propriété intellectuelle dans leur champ d'activité.

Le standard JPEG-2000 prévoit l'insertion d'informations sur la sécurité dans l'en-tête des fichiers.

La norme MPEG-21 ou ISO/IEC 21000 (21 pour $\mathrm{XXI}^{\mathrm{e}}$ siècle) cherche à développer une infrastructure de diffusion, d'échange et de consommation des contenus multimédias en associant plusieurs types de technologies destinées à tous les intervenants de la chaîne de production et de consommation. MPEG-21 normalise les spécifications de la syntaxe, de la sémantique et des interfaces qui relient de manière sécurisée et persistante différents éléments : déclaration, identification et description du contenu, adaptation aux réseaux et aux conditions techniques de chaque élément de la chaîne de distribution, automatisation de la transaction, rapport d'événements et protection en conformité avec les droits d'utilisation liés au contenu. Le concept original de digital item est une notion composite et évolutive, c'est une unité de transaction qui associe une ressource numérique, des métadonnées et les informations sur la structuration de l'ensemble. Par exemple, une compilation musicale associera des fichiers au format mp3, des photos, des clips vidéo, des liens hypertextes vers une page d'information, etc.

\section{L'expression des conditions juridiques}

d'utilisation des œuvres

Linteropérabilité sémantique de la description des droits portant sur les contenus est obtenue par l'élaboration de dictionnaires et de langages d'expression des droits, fondés sur des référentiels partagés et formalisés de notions et concepts ou ontologies ${ }^{17}$. Le dictionnaire des droits de MPEG-21 (Rights Data Dictionary ou RDD) contient les termes et les définitions des règles s'appliquant à la distribution d'œuvres en ligne. Il est dérivé du projet $<$ indecs $>^{18}$ de métadonnées pour le commerce électronique et les transactions de droits de propriété intellectuelle permettant d'exprimer les conditions d'utilisation assignées aux contenus. Les expressions sémantiques des règles sont décrites en langage naturel dans le RDD et leur syntaxe est traduite en code informatique dans un "langage d'expression des droits » (Rights Expression Language ou REL).

Le dictionnaire définit les composants d'un langage orienté vers le commerce électronique qui représente en XML les conditions d'accès et de paie- 
ment. L'ensemble RDD-REL associé à un dispositif de protection technique a pour objectif l'application automatique des règles et transactions financières portant sur la vente de contenus protégés.

Le langage XrML (eXtensible Rights Markup Language $^{19}$ ) à l'origine du REL de MPEG-21 fait l'objet de brevets logiciels qui pourraient compromettre ${ }^{20}$ le développement d'autres initiatives de DRM, comme le standard dédié aux appareils mobiles OMA (Open Mobile Alliance ${ }^{21}$ ) qui implémente le langage d'expression des droits ODRL (Open Digital Rights Language ${ }^{22}$ ), disponible sous licence libre GNU-GPL.

Ces deux standards, tous deux fondés sur le métalangage XML permettant de baliser une sémantique, ont été proposés de manière concurrente dans la partie relative aux langages de description des droits de MPEG-21. ODRL et XrML ont vocation à s'intégrer dans tout type de système de gestion des droits et proposent une représentation formelle des relations entre les utilisateurs et les titulaires de droits. Les deux modèles sont construits autour de situations contractuelles et intègrent des procédés d'identification et d'authentification, l'octroi de licences sur des contenus à des conditions prédéfinies étant assuré par un tiers de confiance, élément important dans l'architecture des DRMs.

Les missions de service public (consultation en bibliothèque, constitution et exploitation d'archives, réalisation de supports d'enseignement à partir de contenus protégés, etc.) n'ont pas été intégrées dans ces langages de description des droits, appuyés sur les pratiques commerciales plutôt que sur l'équilibre entre droit des auteurs et droits du public. L'étude du vocabulaire utilisé dans ODRL et XrML permet de déceler une sur-représentation des concepts décrivant les conditions contractuelles de répartition des droits patrimoniaux par rapport aux utilisateurs finals et aux situations en dehors des droits exclusifs.

Le langage d'expression des droits Creative Commons décrit au format rdf les droits des utilisateurs et respecte les exceptions légales aux droits exclusifs. Il permet d'exprimer non pas ce qui est interdit, mais les utilisations qui sont autorisées. Cognitivement et politiquement orientés vers les libertés concédées au public, les contrats Creative Commons prohibent l'utilisation d'une mesure technique de protection.

Il apparaît indispensable de développer un langage qui permettra la communication et la négociation entre les parties à l'échange, et qui régira à la fois les transactions économiques et les utilisations non gouvernées, accordées librement par la loi et le contrat. Les langages actuels sont conçus pour associer à l'œuvre, dès sa création, les conditions juridiques de son utilisation. Pourtant, ces conditions ne peuvent pas être définies totalement à l'avance par les titulaires de droits. Au contraire, nous pensons que l'expression des conditions d'utilisation se construit au fur et à mesure de la circulation sur les réseaux, en fonction non seulement de l'œuvre et des condi- tions déterminées à l'avance par le titulaire des droits, mais également en fonction de la législation nationale, de l'utilisateur et du type d'utilisation. Un tel langage devra être extensible et sous licence libre, de manière à ce que chaque communauté puisse faire évoluer le modèle et ajouter de nouvelles expressions.

Le Digital Media Project (DMP ${ }^{23}$ ) constitue une autre initiative majeure de normalisation, créée par Leonardo Chiariglione, le fondateur de MPEG, en vue de proposer un standard réellement interopérable. À la différence des autres DRMs, DMP prévoit de joindre à ses spécifications une annexe informative décrivant les droits et usages traditionnels de tous les utilisateurs et acteurs de la chaîne de valeur. Cette initiative devrait permettre aux intégrateurs de proposer des DRMs équitables respectant les libertés et les droits individuels.

\section{Conclusion}

Il est souhaitable que des solutions techniques équitables et transparentes soient développées rapidement de manière à éviter les situations de blocage : « La Bibliothèque nationale de France ne devrait pas avoir 15 millions de pages en attente qu'elle ne se risque pas à numériser, faute d'être assurée de ses droits ${ }^{24}$. " Face à la complexité des règles, source d'insécurité juridique, des informations claires (compréhensibles par les non-juristes) sur le régime juridique des œuvres, associées à des interfaces cognitives (utilisables par les non-informaticiens) pour l'expression, la négociation et la gestion des droits, sont indispensables. Des fonctions d'insertion de métadonnées bibliographiques et juridiques peuvent être intégrées dans les logiciels d'édition, les plates-formes de publications, les moteurs de recherche, afin de faciliter l'accès au droit et l'accès aux œuvres.

\section{D. de $\mathbf{R}$.}

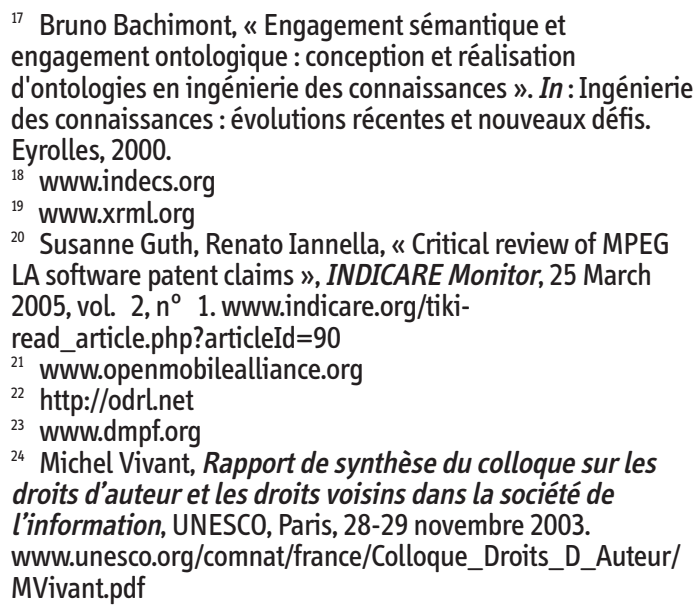

